\title{
Dural Arteriovenous Fistula on the Brain Stem and Upper Cervical Spinal Cord
}

\author{
- A Case Report -
}

\author{
Hyung-Suk Sun, M.D., Hyun-Sik Yun, M.D., Min-Keun Song, M.D., \\ Jae-Young Han, M.D., In-Sung Choi, M.D. Ph.D., Sam-Gyu Lee, M.D., Ph.D.
}

Department of Physical \& Rehabilitation Medicine, Research Institute of Medical Sciences, Center for Aging and Geriatrics, Regional CardioCerebrovascular Center, Chonnam National University Medical School \& Hospital, Gwangju 501-757, Korea

\begin{abstract}
A 53-year-old man abruptly developed headache and unconsciousness. Brain computed tomography (CT) and CT angiography showed subarachnoid hemorrhage, intraventricular hemorrhage, and multiple tortuous vascular structures on the brain stem and upper cervical spinal cord. Four-vessel angiography displayed intradural ventral arteriovenous fistula, supplied by the left vertebral and occipital arteries. Drainage was via both sigmoid sinus and cervical venous plexus. He had been treated with transarterial coil embolization of the left vertebral artery. Subsequently, he suffered from left hemiplegia and cognitive problem. Brain magnetic resonance (MR) and MR angiography performed 4 weeks later revealed multiple infarctions on the left cerebellum, left upper cervical spinal cord, and both medial thalamus, as well as occlusion of the left vertebral artery with reduction in varix size. After rehabilitative management, his muscle strength and cognitive function improved. We report a very rare case of dural arteriovenous fistula on the brain stem and upper cervical spinal cord.
\end{abstract}

Key Words Arteriovenous fistula, Brain stem, Spinal cord

\section{INTRODUCTION}

Dural arteriovenous fistula (DAVF) is caused by an abnormal arteriovenous shunt in the dura mater of the brain. DAVF are located within or near the wall of the

Received April 22, 2010; Accepted November 29, 2010

Corresponding author: Sam-Gyu Lee

Department of Physical \& Rehabilitation Medicine, Chonnam National University Medical School \& Hospital, 8, Hak-dong, Dong-gu, Gwangju 501-757, Korea

Tel: +82-62-220-5198, Fax: +82-62-228-5975, E-mail: LEE9299@hitel.net (c) This is an open-access article distributed under the terms of the Creative Commons Attribution Non-Commercial License (http:// creativecommons.org/licenses/by-nc/3.0) which permits unrestricted noncommercial use, distribution, and reproduction in any medium, provided the original work is properly cited.

Copyright $\odot 2011$ by Korean Academy of Rehabilitation Medicine dural venous sinus, and most frequently found in the cavernous sinus, transverse sinus or sigmoid sinus. ${ }^{1}$ These acquired lesions often occur after suffering dural sinus thrombosis ${ }^{2}$ and account for $10-15 \%$ of all intracranial vascular malformations. People aged 40-60 are generally more prone to suffering from them. ${ }^{1}$

Spinal DAVF accounts for about $70 \%$ of all spinal arteriovenous malformations (AVMs) and is the most frequent disease among all spinal vascular diseases. ${ }^{3}$ Middle-aged men are prone to spinal DAVF, and lesions are likely to occur around the dorsal surface of thoracolumbar spinal cord. Clinical symptoms are those of progressive myelopathy such as spastic paraplegia, sensory disturbance, bladder dysfunction, and bowel 
dysfunction, and it is rare that hemorrhage accompanies it. ${ }^{4}$

This study reports with literature review a very rare DAVF case where the lesion was located on the brain stem and upper cervical spinal cord.

\section{CASE REPORT}

A 53-year-old man with major complaints of a sudden onset of headache and mental deterioration came to the emergency center of the hospital. On admission, he was
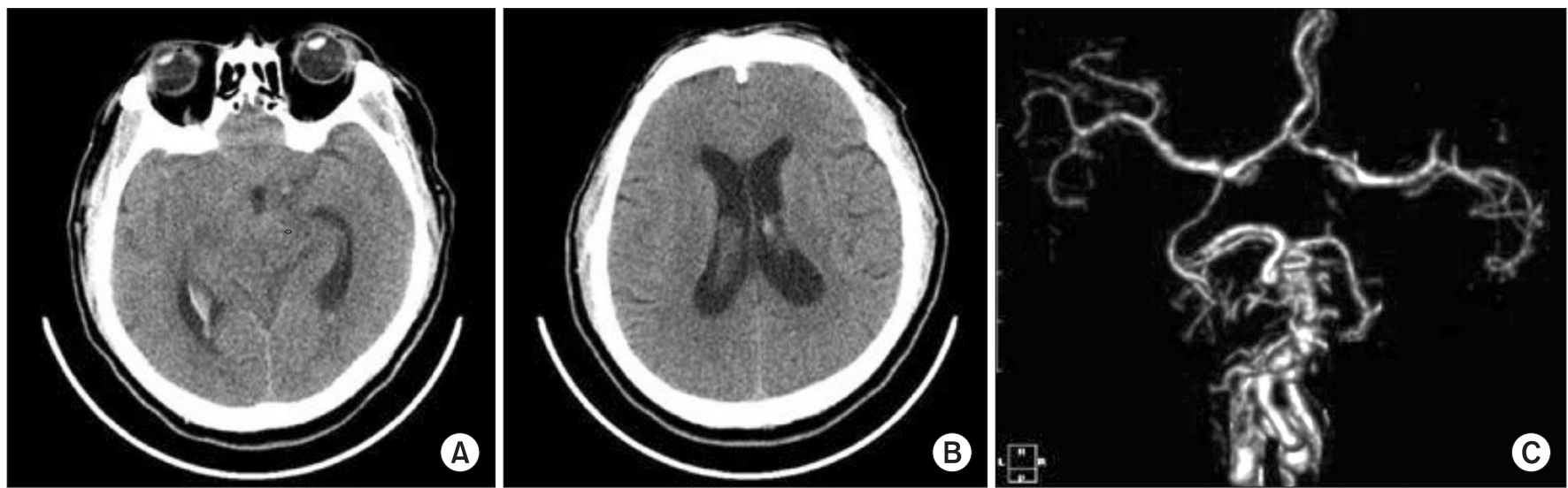

Fig. 1. Brain CT and CT angiography show diffuse subarachnoid hemorrhage, intraventricular hemorrhage with hydrocephalus (A, B), and tortuous vascular structures along anterior aspect of brain stem and upper cervical spinal cord (C).
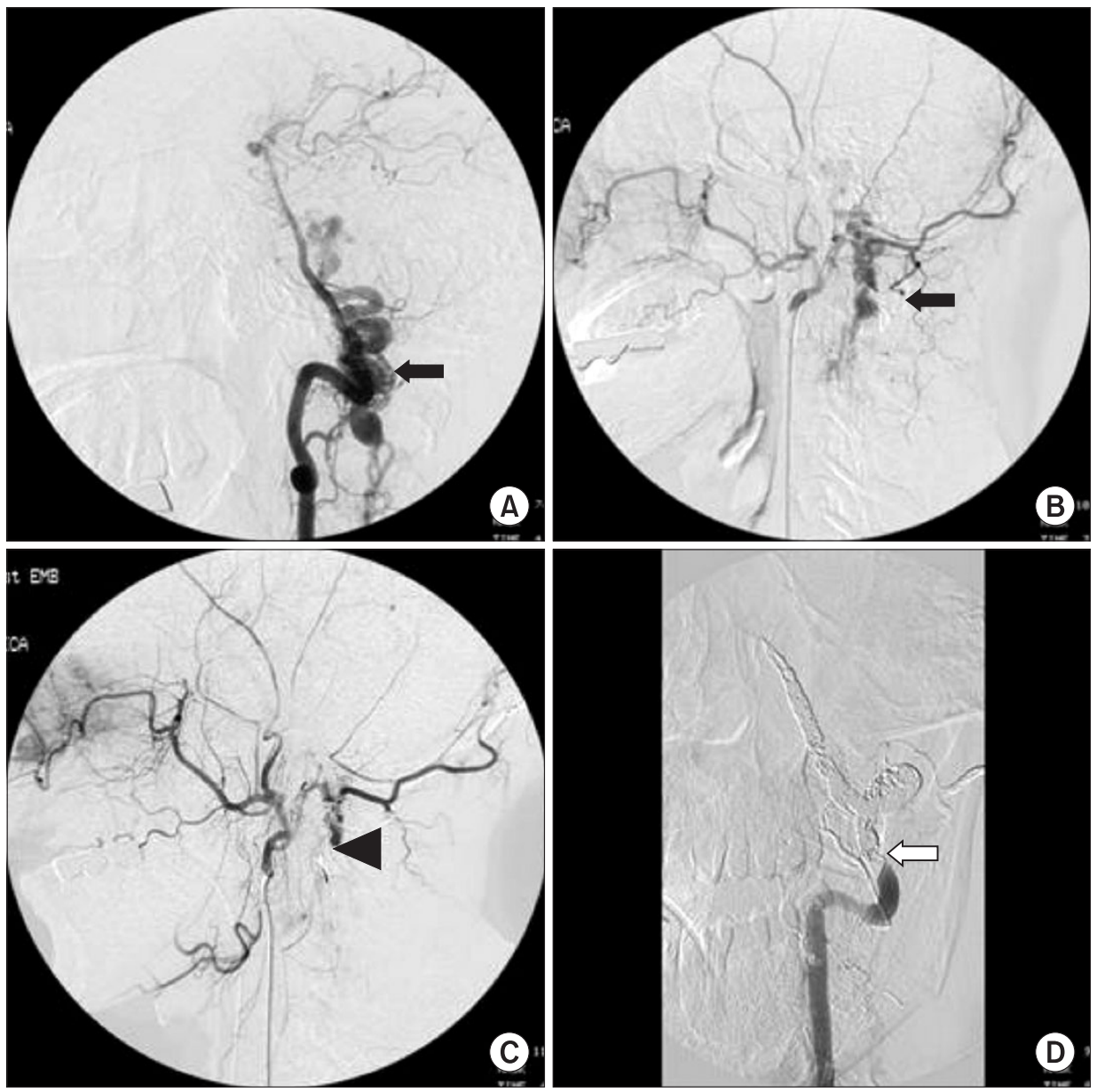

Fig. 2. Cerebral angiography shows dural arteriovenous fistula supplied by the left vertebral and occipital arteries (black arrow) (A, B). Transarterial embolization through the left occipital artery failed (black arrow head) (C). However, the left vertebral artery was super-selected with microwire and microcatheter and was embolized with embolic materials, resulting in partial occlusion (white arrow) (D). 
drowsy and his Glasgow Coma Scale (GCS) score was 14 , and no focal neurologic signs were detected. He had no medical history of diabetes mellitus, hypertension, tuberculosis, and hepatitis, and as for family history, his mother had suffered from cerebral hemorrhage.

Brain computed tomography (CT) and CT angiography carried out on admission showed a subarachnoid hemorrhage (SAH), intraventricular hemorrhage (IVH) with hydrocephalus, and a tortuous vascular structure that was observed around the brain stem and upper cervical spinal cord (Fig. 1). Cerebral angiography was performed when vital signs had been stabilized and headache had been controlled through conservative treatments. The cerebral angiogram on the left vertebral artery revealed multiple vascular structures around the V4 segment of the left vertebral artery, which were drained to both sides of the sigmoid sinus and vertebral venous plexus. A cerebral angiogram on the external carotid artery showed multiple vascular structures around the occipital artery, which were connected to the drained vein observed via the left vertebral artery angiogram. Embolization was attempted on the left occipital artery and the distal V2 segment of the left vertebral artery, but the treatment was halted due to reflux. Another embolization was performed from the V4 and V3 segment to some of the distal V2 segment of the left vertebral artery. The treatment was terminated after partial occlusion was identified (Fig. 2 ). As mental deterioration and left hemiplegia were observed after the embolization, cerebral angiography and brain CT were conducted. However, new lesions such as cerebral hemorrhages or vascular occlusions were not found. One week after the embolization, followup brain CT displayed subacute cerebral infarction on the left cerebellum and thalamus, and a mild mass effect on the fourth ventricle and brain stem. The patient still felt drowsy and scored 14 on the GCS after being transferred to the department of rehabilitation medicine. Manual muscle tests of the left upper and lower limbs were measured as medical research council (MRC) grade 2 and hypoesthesia of the left face and hemibody was found. The patient also complained of dysarthria and diplopia. Spasticity, bladder dysfunction, bowel dysfunction, or dyspnea was not noticed. The patient scored 6 on the Korean-modified Barthel index (K-MBI), and the functional independence measure (FIM) score was 40 . He was able to move in a wheel chair, fully dependent on a caregiver. Brain magnetic resonance image (MRI) and MR angiography performed one month after the onset showed infarctions on the left cerebellum, medial thalami, and the left posterolateral side of the upper cervical spinal cord. The occlusion of the left vertebral artery, as well as reduction in vascular structures that had been located on the brain stem and upper cervical spinal cord, were detected (Fig. 3). At that time, somatosensory evoked potential (SSEP) obtained by stimulating the right median nerve and motor evoked potential (MEP) recorded from the right abductor pollicis brevis and abductor hallucis were normal. However,
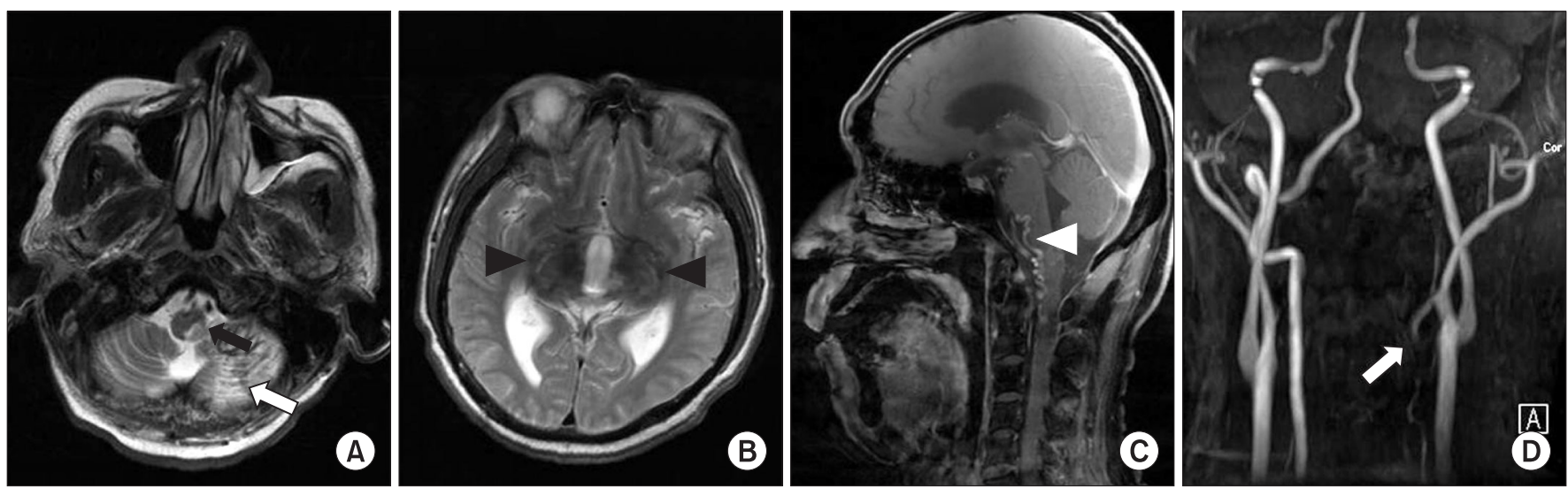

Fig. 3. Axial T2-weighted images of Brain MRI show subacute to chronic hemorrhagic infarction involving left cerebellar hemisphere (white arrow), left posterolateral aspect of upper cervical spinal cord (black arrow) (A), and both medial thalamus (black arrow head) (B). Sagittal gadolinium enhanced image shows reduction of tortuous vascular structure (white arrow head) (C). MR angiography shows occlusion of left vertebral artery (white arrow) (D). 
SSEP of the left median nerve and MEP from the left abductor pollicis brevis were not evoked, while the latency of MEP recorded from the left abductor hallucis was delayed. The patient was put on anti-thrombotic drugs and medications for improving cognitive function, while continuing to take active assisted range of motion exercise, Bobath neurodevelopmental treatment, mat exercise, and occupational therapy. Six months after the onset, he recovered enough to bring his Korean MiniMental Status Examination (K-MMSE) score to 21 points, while manual muscle tests of the left upper limb were grade 3, and those of left lower limb corresponded to grade $3-4$. The patient showed a significant recovery by scoring 73 on the K-MBI and 86 on the FIM. He was able to take gait training with minimum dependence on a caregiver.

\section{DISCUSSION}

About $2 \%$ of DAVF occur around the cervical spine, and in most cases they take form of myelopathy. ${ }^{5}$ It is very rare that lesions are located on the brain stem and upper cervical spinal cord, and there have been reports that such case accompany the symptoms of progressive myelopathy. ${ }^{6}$ It has been considered that spinal DAVF drained upward or inside the cranium are often related to $\mathrm{SAH},{ }^{7}$ and venous hypertension is thought to cause it although the exact mechanism has not been confirmed to date. ${ }^{8}$

The patient in this case had initial symptoms of SAH and IVH such as headache and mental deterioration. Through a cerebral angiography, a DAVF was diagnosed, which was fed by the left vertebral artery and occipital artery, and drained to both sides of the sigmoid sinus and vertebral artery plexus. According to the classification of Spetzler et al., this case corresponded to the intradural ventral arteriovenous fistula. ${ }^{9}$ When the causes of intracranial SAH are not found on an initial cerebral angiography, spinal DAVF fed by right vertebral artery should be considered. ${ }^{8}$ Delayed diagnosis of DAVF had a possibility to worsen its prognosis. However, the vascular malformation of our patient was located around the brain stem and upper cervical spinal cord and was large enough to be detected. In addition, recent initial cerebral angiographic protocol includes bilateral vertebral arteries, making it relatively easy to diagnose DAVF.
There have been various reports on incidence and mortality rates of DAVF in the upper cervical spinal cord. Ricolfi et al. ${ }^{10}$ reported that patients with intracranial DAVFs accompanied by venous drainage around the medulla oblongata tend to have symptoms of paraplegia or tetraplegia, sensory disturbance, and anal sphincter dysfunction. Among five cases reported, two patients completely recovered, one showed considerable improvement, and two died. ${ }^{10}$ Aviv et al. ${ }^{7}$ described that $45 \%$ of 44 cervical DAVF patients presented with SAH, $43 \%$ of them presented with myelopathy. Other symptoms such as radiculopathy, 6th cranial nerve palsy were also reported. ${ }^{7}$ Among two cervical DAVF patients who had headache and mental deterioration without focal neurological deficits, one died of shunt-related complications before planned surgical treatment was performed, while the other made good recovery after surgical removal of cervical DAVF supplied by right vertebral artery. ${ }^{7}$ As DAVF has rarely been reported to be located on the brain stem and upper cervical spinal cord, forecasting its prognosis is very difficult.

The patient in this case was affected by left hemiplegia after undergoing the embolization, but drug treatment and rehabilitation were continued, as the hemiplegia was diagnosed early. The left vertebral artery was occluded after the embolization, reducing the size of the tortuous vascular structures. We believe that areas affected by the infarctions had little influence on motor functions, and therefore a significant functional recovery was seen six months after the onset.

We report a DAVF case with literature review, where the DAVF was located on the brain stem and upper cervical spinal cord, which has rarely been considered as a cause for SAH and IVH.

\section{ACKNOWLEDGEMENTS}

This study was supported by a grant of the Korea Healthcare technology R\&D Project, Ministry for Health, Welfare \& Family Affairs, Republic of Korea (A084869).

\section{REFERENCES}

1. Malek AM, Halbach VV, Higashida RT, Phatouros CC, Meyers PM, Dowd CF. Treatment of dural arteriovenous malformations and fistulas. Neurosurg Clin $\mathrm{N}$ 
Am 2000; 11: 147-166

2. Kan P, Stevens EA, Warner J, Couldwell WT. Resolution of an anterior-inferior cerebellar artery feeding aneurysm with the treatment of a transverse-sigmoid dural arteriovenous fistula. Skull Base 2007; 17: 205210

3. Gilbertson JR, Miller GM, Goldman MS, Marsh WR. Spinal dural arteriovenous fistulas: MR and myelographic findings. Am J Neuroradiol 1995; 16: 20492057

4. Rosenblum B, Oldfield EH, Doppman JL, Di Chiro G. Spinal arteriovenous malformation: a comparison of dural arteriovenous fistulas and intradural AVM's in 81 patients. J Neurosurg 1987; 67: 795-802

5. Kohno M, Takahashi H, Ide K, Ishijima B, Yamada K, Nemoto S. A cervical dural arteriovenous fistula in a patient presenting with radiculopathy. J Neurosurg 1997; 84: 119-123

6. Li J, Ezura M, Takahashi A, Yoshimoto T. Intracranial dural arteriovenous fistula with venous reflux to the brainstem and spinal cord mimicking brainstem infarction-case report. Neurol Med Chir 2004; 44: 2428

7. Aviv RI, Shad A, Tomlinson G, Niemann D, Teddy PJ, Molyneux AJ, Byrine JV. Cervical dural arteriovenous fistulae manifesting as subarachnoid hemorrhage: report of two cases and literature review. Am J Neuroradiol 2004; 25: 854-858

8. Do HM, Jensen ME, Cloft HJ, Kallmes DF, Dion JE. Dural arteriovenous fistula of the cervical spine presenting with subarachnoid hemorrhage. Am J Neuroradiol 1999; 20: 348-350

9. Spetzler RF, Detwiler PW, Riina HA, Porter RW. Modified classification of spinal cord vascular lesions. J Neurosurg 2002; 96 Suppl 2: 145-156

10. Ricolfi F, Manelfe C, Meder JF, Arrué P, Decq P, Brugiées P, Cognard C, Gaston A. Intracranial dural arteriovenous fistulae with perimedullary venous drainage. Anatomical, clinical and therapeutic considerations. Neuroradiology 1999; 41: 803-812 